\title{
Fraktionsausschluss. Zum Urteil des Verfassungsgerichtshofs Rheinland-Pfalz vom 29. Januar 2019 - VGH O 18/18
}

\author{
Siegfried Jutzi
}

Partei- und Fraktionsausschlussverfahren wird es wohl immer geben (müssen). Jüngere Parteien und „ihre“ Fraktionen dürften davon stärker betroffen sein als etablierte, da sie sich intensiver um die Strukturierung ihres Profils in sachlicher und personeller Hinsicht bemühen müssen. Angesichts eines tendenziellen Einflussverlustes so genannter großer Volksparteien, wodurch Repräsentationslücken für neue politische Gruppierungen eröffnet werden ${ }^{1}$, sind weitere Verfahren zu erwarten. Die Entscheidung des VerfGH Rheinland-Pfalz, die in diesem Umfeld angesiedelt ist, verdient allein deswegen Beachtung. Es kommt hinzu, dass sie die bisherige verfassungsgerichtliche Rechtsprechung und Literatur strukturiert aufbereitet und Umfang wie Intensität der verfassungsrechtlichen Überprüfung fortentwickelt.

\section{Sachverhalt}

Der Antragsteller, Mitglied der politischen Partei AfD (Alternative für Deutschland), wandte sich als Abgeordneter des Landtags Rheinland-Pfalz im Organstreitverfahren vor dem VerfGH Rheinland-Pfalz gegen den Ausschluss aus seiner Fraktion. Der Ausschluss war im September 2018 durch Beschluss der Fraktionsversammlung gestützt auf die Fraktionssatzung und -geschäftsordnung erfolgt. Danach kann die Fraktionsversammlung bei Vorliegen eines wichtigen Grundes in geheimer Abstimmung unter Beachtung näherer Bestimmungen über das Verfahren den Ausschluss von Mitgliedern aus der Fraktion mit Zweidrittelmehrheit beschließen. Als wichtiger Grund wird angesehen, wenn ein Mitglied gegen die freiheitlich-demokratische Grundordnung verstößt, dem Ansehen der Fraktion großen Schaden zufügt, seinen in dieser Satzung aufgeführten Pflichten beharrlich nicht nachkommt, aus der Partei ausgetreten ist oder ausgeschlossen wurde.

Die antragsgegnerische Fraktion hatte den Ausschluss damit begründet, der Antragsteller habe das Vertrauensverhältnis zur Fraktion zerstört und dieser in der Öffentlichkeit großen Schaden zugefügt. In tatsächlicher Hinsicht wurde dies darauf gestützt, dass der Antragsteller punktuell mit der extremistischen Szene zusammengearbeitet habe, wie etwa durch einen Vortrag auf einer Veranstaltung, für die ein anwesendes ehemaliges Mitglied der NPD geworben habe. Mit diesem habe der Antragsteller auch in einem WhatsAppChat kommuniziert. Der Antragssteller war wegen dieser Vorfälle durch den Landesverband AfD Rheinland-Pfalz zuvor zweimal - am 15. Juni und 7. September 2018 - abgemahnt worden. Dabei wurde ihm auch vorgehalten, er habe gegenüber dem Landesverband falsche Aussagen gemacht und tatsächliche Gegebenheiten verschleiert beziehungsweise verschwiegen, was der Partei erheblichen Schaden zugefügt habe.

Am 12. September 2018 beantragte der Fraktionsvorstand in einem an alle Fraktionsmitglieder gerichteten Schreiben, den Antragsteller mit sofortiger Wirkung aus der Fraktion auszuschließen und begründete dies näher im Wesentlichen damit, der Antragsteller sei vom

1 Vgl. Werner J. Patzelt, Mangel in der Responsivität oder Störung in der Kommunikation? Deutschlands Repräsentationslücke und die AfD, in: ZParl, 49. Jg. (2018), H. 2, S. 885 - 895. 
Fraktionsvorstand mehrfach zum persönlichen Gespräch geladen und seitens des Landesvorstands zweimal schriftlich abgemahnt worden. In der Fraktionssitzung am 5. September 2018 habe er erneut bestritten, persönliche Kontakte in die extremistische Szene zu haben. Nunmehr lägen dem Fraktionsvorstand jedoch eindeutige Beweise vor, welche die Unwahrheit der Aussagen belegten. Am 15. September 2018 lud der Parlamentarische Geschäftsführer der Fraktion zu einer außerordentlichen Fraktionssitzung am 18. September 2018, in der über den Antrag des Fraktionsvorstands beraten und entschieden werden sollte. An der Sitzung nahm der Antragsteller teil und kam auch zu Wort. In geheimer Abstimmung wurde er schließlich mit Zweidrittelmehrheit aus der Fraktion ausgeschlossen.

Mit der Organklage ${ }^{2}$ rügte der Antragsteller, der Fraktionsausschluss verstoße gegen seine organschaftlichen Rechte aus Art. 79 Abs. 2 und Art. 85a Abs. 1 Satz 1 der Verfassung für Rheinland-Pfalz (LV RP). Weder sei er ordnungsgemäß angehört noch seien ihm die Vorwürfe hinreichend mitgeteilt worden, auch rechtfertigen sie nicht seinen Faktionsausschluss.

Die Fraktion als Antragsgegnerin verteidigte diesen unter Vorlage diverser Unterlagen und wies insbesondere darauf hin, dass der Verhandlungsgegenstand der maßgeblichen Fraktionsversammlung in vollem Umfang für sämtliche Fraktionsmitglieder erkennbar und hinreichend bestimmt gewesen sei. Der Antragsteller sei mehrfach auf Partei- und auf Fraktionsebene mit den Vorwürfen konfrontiert worden und habe auf der Fraktionsversammlung die Möglichkeit zur Stellungnahme gehabt. Die belastenden Unterlagen hätten zwischen der Antragstellung und der Fraktionssitzung im Büro des Fraktionsvorsitzenden zur Einsicht ausgelegen. Sachlich sei der Fraktionsausschluss wegen der Kooperation des Antragstellers mit politisch für die Fraktion untragbaren Personen gerechtfertigt.

\section{Wesentliche Entscheidungsgründe}

\subsection{Zulässigkeit des Organstreits}

Der VerfGH stellt fest, der gegen eine Landtagsfraktion gerichtete Antrag eines aus ihr ausgeschlossenen Abgeordneten könne Gegenstand eines Organstreitverfahrens nach Art. 130 Abs. 1 LV RP, $\$ 2$ Nr. 1 a, $\$ \$ 23$ ff. VerfGHG RP sein. Der Antragsteller sei als Abgeordneter des Landtags als „anderer Beteiligter“ im Sinne von Art. 130 Abs. 1 Satz 2 LV antragsberechtigt und, soweit er eine Verletzung seiner in Art. 79 Abs. 2 und Art. 85a Abs. 1 Satz 1 LV RP gewährleisteten Rechte rügt, auch antragsbefugt.

\subsection{Begründetheit des Organstreits (verfassungsrechtliche Maßstäbe)}

In der Sache wies der VerfGH den Antrag als unbegründet zurück. Die Fraktion habe durch den Ausschluss des Antragstellers dessen aus dem Statusrecht eines Abgeordneten folgenden Anspruch aus Art. 79 Abs. 2, Art. 85a Abs. 1 Satz 1 LV RP auf willkürfreie Entscheidung nicht verletzt.

(1) Den Anfang der Begründetheitsprüfung bilden recht umfangreiche eigene und Entscheidungen anderer Verfassungsgerichte sowie wissenschaftliche Stellungnahmen berück-

2 Ein parallel gestellter Antrag auf Erlass einer einstweiligen Anordnung, die Vollziehung des Fraktionsausschlusses einstweilen auszusetzen, war vom Verfassungsgerichtshof Rheinland-Pfalz mit Beschluss vom 5. November 2018 - VGH A 19/18 - (juris) abgelehnt worden. 
sichtigende Ausführungen zur Bedeutung der Fraktionen im Parlament, der Stellung der ihnen angehörenden Abgeordneten sowie der sich aus diesem Spannungsverhältnis ableitenden verfahrens- und materiell-rechtlichen Anforderungen an einen Fraktionsausschluss.

Bezogen auf Fraktionen stellt der VerfGH heraus, sie seien „für das Verfassungsleben notwendige und zugleich die das Parlament bestimmenden Einrichtungen, denen von Verfassungs wegen das Recht zur Mitwirkung an der Erfüllung der Aufgaben des Landtags garantiert sei (siehe Art. 85a Abs. 2 Satz 1 LV)“. Sie organisierten das parlamentarische Geschehen arbeitsteilig und sicherten die parlamentarische Funktionsfähigkeit vor allem durch mehrheitsfähige Meinungsbündelung.

Die Möglichkeit, eine Fraktion zu bilden und in ihr mitzuarbeiten, verändere die Wirkungsmöglichkeiten des einzelnen Abgeordneten daher nicht unerheblich. ${ }^{3}$ Die Bildung und Mitarbeit in einer Fraktion habe eine gewichtige Bedeutung bei der Ausübung des Abgeordnetenmandats. ${ }^{4}$

Sodann betont der VerfGH, die Bildung einer Fraktion beruhe auf der jedem einzelnen Abgeordneten gewährleisteten Ausübung des freien Mandats. Dies umfasse die Befugnis, sich mit anderen Abgeordneten zu einer Fraktion zusammenzuschließen und mit ihnen zusammenzubleiben ${ }^{5}$, ebenso wie die Befugnis der übrigen Fraktionsmitglieder, einen einzelnen Abgeordneten grundsätzlich aus ihren Reihen ausschließen zu können. ${ }^{6}$ Das Statusrecht des Abgeordneten und das daraus folgende Fraktionsbildungsrecht seien damit Grund und Grenze der Entscheidung der Fraktion über den Ausschluss eines ihrer Mitglieder. Aus den verfassungsrechtlich geschützten Belangen der Fraktion und ihrer Funktion für einen effektiven Parlamentsbetrieb ergebe sich eine verfassungsrechtliche Grenze für die Rechte der einzelnen Abgeordneten. ${ }^{7}$

Der Fraktionsausschluss setzt danach ein rechtsstaatlichen Mindestanforderungen genügendes Verfahren sowie einen willkürfreien Entschluss der Fraktionsversammlung voraus.

(2) $\mathrm{Zu}$ den sich aus dem Rechtsstaatsprinzip und dem Statusrecht des Abgeordneten ergebenden verfahrensrechtlichen Anforderungen ${ }^{8}$ nennt der VerfGH insbesondere die $\mathrm{Zu}$ -

3 Unter Hinweis auf BVerfGE 43, S. 142, S. 149; Verfassungsgericht Brandenburg, in: NVwZ-RR 1997, S. 577, S. 578; Volker Perne, in: Lars Brocker / Michael Droege / Siegfried Jutzi, Verfassung für Rheinland-Pfalz, Kommentar, Baden-Baden 2014, Art. 85a Rn. 12.

4 Unter Hinweis auf Verfassungsgerichtshof Berlin, in: NVwZ-RR 2005, S. 753, S. 754.

5 Unter Hinweis unter anderem auf BVerfGE 140, S. 1, S. 31 Rn. 86; BVerfGE 142, S. 25, S. 61 Rn. 97; Hans Hugo Klein, Austritt, Ausschluss, Rechte: Der fraktionslose Abgeordnete. Eine Veranstaltung der Deutschen Vereinigung für Parlamentsfragen - Vortrag I, in: ZParl, 35. Jg. (2004), H. 4, S. 627 - 632, S. 627, S. 629; betreffend die Abwahl aus einem Ausschuss Verfassungsgerichtshof Baden-Württemberg, in: NVwZ-RR 2018, S. 129, S. 130.

6 Unter Hinweis auf Landesverfassungsgericht Mecklenburg-Vorpommern, in: DÖV 2003, S. 765, S. 767; Christofer Lenz, Der Fraktionsausschluss - Zwischenbilanz nach den Fällen Möllemann und Hohmann, in: NVwZ, 24. Jg. (2005), H. 4, S. 364 - 370, S. 364, S. 365; Lars Brocker 1 Volker Perne, Parlamentsrecht im Wandel. Die Entwicklung des Parlamentsrechts im Landtag Rheinland-Pfalz in der 15. Wahlperiode (2006-2011), Teil I: Geschäftsordnung, in: LKRZ, 5. Jg. (2011), H. 5, S. 161 - 166, S. 161, S. 165.

7 Unter Hinweis auf Verfassungsgerichtshof Berlin, in: DVBl. 2018, S. 1287, S. 1288; betreffend die Abwahl aus einem Ausschuss Verfassungsgerichtshof Baden-Württemberg, in: NVwZ-RR 2018, S. 129, S. 130; Hans Hugo Klein, a.a.O. (Fn. 5), S. 627, S. 630.

8 Dazu in Bezug auf Art. 38 Abs. 1 Satz 2 GG Lars Brocker, in: Wolfgang Kahl / Christian Waldhoff / Christian Walter (Hrsg.), Bonner Kommentar zum Grundgesetz, Loseblatt, (150. EL Februar 2011), Art. 40 Rn. 193. 
ständigkeit der Fraktionsversammlung für die Entscheidung über den Fraktionsausschluss sowie ein Ausschlussverfahren, das dem betroffenen Abgeordneten hinreichend Gelegenheit zur wirksamen Stellungnahme einräumt und den Fraktionsmitgliedern die Möglichkeit gibt, diese zu berücksichtigen und an der Entscheidung verantwortlich mitzuwirken. Dies erfordert die schriftliche Mitteilung des Ausschlussantrags und der geltend gemachten Gründe, eine angemessene Vorbereitungszeit für alle Beteiligten, die Einberufung der Fraktionsversammlung, die Abstimmung und die erforderliche Mehrheit bei der Beschlussfassung.

Das verbreitete Quorum einer Zweidrittelmehrheit der Mitglieder sieht der VerfGH grundsätzlich als unbedenklich an. ${ }^{9}$ Ob dieses Quorum auch verfassungsrechtlich geboten ist, wofür nach Auffassung des VerfGH einiges spricht, lässt der Gerichtshof mangels Entscheidungserheblichkeit offen, und stellt fest, dass die genannten formellen Voraussetzungen eines Fraktionsausschlusses mangels Wertungsbedürftigkeit der uneingeschränkten verfassungsgerichtlichen Überprüfung unterliegen.

(3) Hinsichtlich der materiellen Voraussetzungen folgt der VerfGH im Kern der ganz überwiegenden Auffassung, wonach das Vorliegen eines qualifizierten - „(besonders) wichtigen " - Grundes verlangt wird. ${ }^{10}$ Als solcher komme nur ein Verhalten in Betracht, das die wesentlichen Grundlagen und Ziele der Fraktion nachhaltig beeinträchtige.

Davon ausgehend bejaht der VerfGH einen wichtigen Grund für einen Fraktionsausschluss insbesondere dann, ,wenn das für eine sinnvolle Meinungsbildung und Arbeit der Fraktion erforderliche Mindestmaß an prinzipieller politischer Übereinstimmung fehlt oder wenn das Fraktionsmitglied das Vertrauensverhältnis so nachhaltig gestört hat, dass den anderen Fraktionsmitgliedern die weitere Zusammenarbeit nicht mehr zugemutet werden kann, oder wenn ein Fraktionsmitglied durch sein Verhalten das Ansehen der Fraktion in der Öffentlichkeit nachhaltig schädigt und die Außenwirkung der Fraktion und deren Wirkungsmöglichkeiten damit beeinträchtigt ${ }^{\text {"11 }}$. Die durch einen Fraktionsausschluss im Einzelfall konkret zu schützenden Belange seien ganz wesentlich von den Fraktionsmitgliedern selbst zu bestimmen. Gleiches gelte, soweit es um die Beurteilung gehe, wann ein schwerer politischer Schaden für die Fraktion vorliege. Die Fraktionsautonomie beanspruche nicht nur gegenüber Dritten Geltung, sondern sei als „innere Fraktionsautonomie“ auch innerhalb des Parlaments und innerhalb der Fraktion selbst zu beachten. ${ }^{12}$ Die gerichtliche Kontrolle der Ausschlussentscheidung habe ebenfalls die fraktionseigenen Wertungen zu achten und der Fraktion einen erheblichen Entscheidungsspielraum zu belassen. ${ }^{13}$

9 Unter Hinweis auf Sven Hölscheidt, Das Recht der Parlamentsfraktionen, Rheinbreitbach 2001, S. 476 ff.; Martin Morlok, Austritt, Ausschluss, Rechte: Der fraktionslose Abgeordnete. Eine Veranstaltung der Deutschen Vereinigung für Parlamentsfragen - Vortrag II, in: ZParl, 35. Jg. (2004), H. 4, S. 633 - 645, S. 633, S. 644; Christofer Lenz, a.a.O. (Fn. 6), S. 364, S. 367 f.

10 Unter Hinweis auf Landesverfassungsgericht Mecklenburg-Vorpommern, in: DÖV 2003, S. 765, S. 768; Verfassungsgerichtshof Berlin, in: NVwZ-RR 2006, S. 441, S. 443; Verfassungsgerichtshof Berlin, in: DVBl. 2018, S. 1287, S. 1288 f.; Peter Badura, in: Wolfgang Kahl / Christian Waldhoff / Christian Walter (Hrsg.), Bonner Kommentar zum Grundgesetz, Loseblatt (193. EL Oktober 2018), Art. 38 Rn. 92; Christofer Lenz, a.a.O. (Fn. 6), S. 364, S. 368.

11 Verfassungsgerichtshof Rheinland-Pfalz, Urteil vom 29. Januar 2019 - VGH O 18/18 - (juris), Leitsatz 2; vgl. auch Rn. 39 f.

12 Unter Hinweis auf Bernd Grzeszick, Fraktionsautonomie als Teil des verfassungsrechtlichen Status der Bundestagsfraktionen, in: NVwZ, 36. Jg. (2017), H. 14, S. 985 - 992, S. 985, S. 990.

13 Unter Hinweis u.a. auf Martin Morlok, a.a.O. (Fn. 9), S. 633, S. 644 f.; Alexandra Bäcker, Der Ausschluss aus der Bundestagsfraktion, Berlin 2011, S. 185 mit Fn. 780; BVerfGE 104, S. 310, 
Weiter weist der VerfGH darauf hin, dass ein Fraktionsausschluss keine Strafmaßnahme sei, sondern letztlich einen Akt des Selbstschutzes zur Aufrechterhaltung der effektiven politischen Arbeit der Fraktion darstelle. Ob der Abgeordnete der Fraktion bewusst und gezielt geschadet habe, sei daher irrelevant.

(4) Abschließend befasst sich der VerfGH näher mit dem Prüfungsmaßstab, den er auf eine Willkürkontrolle beschränkt sieht. Ein Fraktionsausschluss sei als letztlich politische Entscheidung verfassungsgerichtlich nicht daraufhin zu überprüfen, ob er vertretbar ist, sondern ob das Statusrecht des Abgeordneten in grundlegender Weise evident verkannt wurde. Diese eingeschränkte Kontrolle erstreckt der VerfGH nicht nur auf die Beurteilung der Frage, ob ein „wichtiger Grund“ für den Ausschluss vorliegt, sondern ausdrücklich auch auf die dieser Entscheidung vorgelagerte Tatsachenebene. Solange und soweit der Entscheidung über den Ausschluss nicht evident unzutreffende tatsächliche Annahmen zu Grunde gelegt worden sind, sieht es der VerfGH nicht als seine Aufgabe, die tatsächlichen Annahmen der Fraktion im Einzelnen durch eigene gegenläufige Annahmen zu ersetzen. ${ }^{14}$ Insbesondere die Bewertung, ob und bei welcher Tatsachendichte bereits eine Störung des Vertrauensverhältnisses innerhalb der Fraktion oder ein Schaden für ihre Öffentlichkeitsdarstellung vorliege, hänge von fraktionsinternen Vorstellungen und Wertungen ab.

\subsection{Begründetheit des Organstreits (Anwendung der Maßstäbe)}

Ausgehend von den genannten Maßstäben gelangt der VerfGH zu dem Ergebnis, dass der Ausschluss des Antragstellers aus der Fraktion sowohl den zu stellenden formellen als auch den materiellen Anforderungen genüge.

(1) Bezogen auf die uneingeschränkt überprüfbaren formellen Voraussetzungen war problematisch, ob der allen Fraktionsmitgliedern schriftlich bekanntzugebende Antrag auch die maßgeblichen Gründe umfasst hatte. Der VerfGH konzediert dem Antragsteller, der an die Fraktionsmitglieder gerichtete Antrag des Fraktionsvorstands sei bei isolierter Betrachtung den an die Darlegung der Ausschlussgründe zu stellenden Anforderungen nicht gerecht geworden. Er lässt es jedoch genügen, dass durch die Bezugnahme auf eine vorherige Fraktionssitzung und auf die zwei Abmahnungen seitens des Landesverbands der AfD das den Fraktionsausschluss begleitende Geschehen jedenfalls erkennbar einen gewissen Niederschlag im Ausschlussantrag gefunden hatte. Außerdem seien die im Antrag (pauschal) gemachten Vorwürfe zuvor Gegenstand der fraktionsinternen Diskussion unter Beteiligung des Antragstellers gewesen. Auch wenn die Abmahnungen als solche nur das Verhältnis des Antragstellers zur Partei betrafen, könnten die sich aus ihnen ergebenden Umstände gleichwohl für die Betrachtung, inwieweit die Vorwürfe schon zuvor Thema auf Fraktionsebene waren, mit herangezogen werden. Trotz der Trennung von Partei und Fraktion spreche grundsätzlich nichts dagegen, dass der Fraktionsvorstand sich diese zu eigen gemacht habe.

(2) Abschließend legt der VerfGH näher dar, dass die Antragsgegnerin in verfassungsrechtlich nicht zu beanstandender Weise die materielle Voraussetzung für einen Fraktionsausschluss, das Vorliegen eines „wichtigen Grundes“, bejaht habe. Auch den Einwand des

S. 332; Verfassungsgerichtshof Berlin, in: NVwZ-RR 2006, S. 441, S. 445; Verfassungsgerichtshof Berlin, in: DVBl. 2018, S. 1287, S. 1289.

14 Anderer Auffassung Christofer Lenz, a.a.O. (Fn. 6), S. 364, S. 370. 
Antragstellers, der Fraktionsausschluss sei unverhältnismäßig, weil er nur als „ultima ratio“ in Betracht komme und vorliegend eine förmliche Abmahnung seitens der Fraktion ausreichend gewesen wäre, weist der VerfGH zurück. Soweit die Fraktion im Rahmen ihres Entscheidungsspielraums in willkürfreier Weise einen „wichtigen Grund“ bejaht habe, sei die Frage nach einem milderen Mittel bereits dorthin vorverlagert. ${ }^{15}$

\section{Stellungnahme}

\subsection{Zulässigkeit des Organstreits}

Der VerfGH geht ohne nähere Begründung davon aus, der gegen eine Landtagsfraktion gerichtete Antrag eines von ihr ausgeschlossenen Abgeordneten könne Gegenstand eines Organstreitverfahrens nach Art. 130 Abs. 1 LV RP, $\$ 2$ Nr. 1 a, $\$ \$ 23$ ff. VerfGHG RP sein. Dem ist im Ergebnis zuzustimmen. Zwar werden nach dem Wortlaut des im Jahr $2000^{16}$ erst geänderten Art. 130 Abs. 1 LV RP lediglich Handlungen eines Verfassungsorgans, nicht aber eines Teils eines Verfassungsorgans erfasst. Jedoch entspricht diese Auslegung eindeutig dem erklärten Willen des Verfassungsgesetzgebers. ${ }^{17}$

\subsection{Verfassungsrechtliche Maßstäbe}

(1) Der VerfGH beschreibt die Rolle der Fraktionen und ihre wichtige Funktion für das Verfassungsleben überzeugend. Daraus zieht er einerseits und zu Recht den Schluss, die Mitarbeit in einer Fraktion habe für die einzelnen Abgeordneten große Bedeutung. Andererseits hegt er keinerlei Zweifel an der grundsätzlichen Berechtigung einer Fraktion, eines ihrer Mitglieder gegen dessen Willen auszuschließen. Trotz ihrer institutionellen Anerkennung in Art. 85a LV RP basiert eine Fraktion als freiwilliger Zusammenschluss von Abgeordneten letztlich auf der freien Entscheidung der sich zur gemeinsamen politischen Arbeit zusammenschließenden Abgeordneten, wurzelt also im Statusrecht der Abgeordneten. ${ }^{18}$ Diese sind frei in ihrer Entscheidung, mit wem und unter welchen Bedingungen sie sich zusammenschließen, aber auch ob sie weiter zusammenbleiben wollen.

Da die Fraktionen jedoch „funktionsnotwendige Organteile des Parlaments“"19, mithin dem staatsorganschaftlichen Bereich zuzurechnen sind ${ }^{20}$, kann ein Fraktionsausschluss nicht im völligen Belieben der Fraktion stehen. Allgemein wird mit gutem Grund ein rechtsstaatlichen Mindestanforderungen genügendes Verfahren sowie eine willkürfreie Entscheidung gefordert.

(2) Den vom VerfGH verlangten verfahrensrechtlichen Mindestanforderungen (Entscheidung durch die Fraktionsversammlung; hinreichend Gelegenheit des Betroffenen zur

15 Unter Hinweis auf Christofer Lenz, a.a.O. (Fn. 6), S. 364, S. 369.

16 GVBl. Rheinland-Pfalz, S. 65.

17 Vgl. dazu Siegfried Jutzi, in: Lars Brocker / Michael Droege / Siegfried Jutzi, a.a.O. (Fn. 3), Art. 130 Rn. 31 mit weiteren Nachweisen.

18 Volker Perne, a.a.O. (Fn. 3), Art. 85a Rn. 1; vgl. auch Paul Georg Schneider, in: Christoph Grimm I Peter Caesar, Verfassung für Rheinland-Pfalz. Kommentar, Baden-Baden 2001; Art. 85a Rn.5; zum GG BVerfGE 140, S. 1, S. 31 Rn. 38; Bernd Grzeszick, a.a.O. (Fn. 12), S. 985 ff.

19 Bernd Grzeszick, a.a.O. (Fn. 12), S. 985, S. 987.

20 Vgl. nur BVerfGE 1, S. 208, S. 225; BVerfGE 80, S. 188, S. 219 f. 
Stellungnahme; ausreichende Information der Fraktionsmitglieder) ist ebenfalls prinzipiell zuzustimmen. Fraglich ist lediglich, ob die vom VerfGH zum Ausdruck gebrachte Sympathie für eine zwingende Zweidrittelmehrheit bei der Beschlussfassung verfassungsrechtlich geboten sein kann.

Allerdings umfasst die Fraktionsautonomie ${ }^{21}$ beziehungsweise die Freiheit der Abgeordneten, sich zu einer Fraktion zusammenzuschließen, die Kompetenz, ein höheres Quorum als zum Beispiel die Mehrheit der Mitglieder in der Fraktionssatzung festzulegen. Fraglich ist indes, woraus sich eine verfassungsrechtliche Verpflichtung ergeben könnte, eine qualifizierte Mehrheit von zwei Dritteln vorzusehen. Das verfassungsrechtlich regelmäßig vorgegebene demokratische Mehrheitsquorum ist entweder die einfache oder die absolute Mehrheit. ${ }^{22}$ Gegen eine relative (einfache) Mehrheit ließe sich einwenden, sie führe zu Zufallsergebnissen. Ebenso wie eine stabile parlamentarische Mehrheit Abweichungen von einer schematischen Anwendung des Mehrheitsprinzips rechtfertigen kann ${ }^{23}$, dürfte dies auch für eine gebildete Fraktion gelten, die in der Regel für eine Wahlperiode konzipiert ist. Dies würde jedoch lediglich die Verpflichtung rechtfertigen, den Fraktionsausschluss von der (absoluten) Mehrheit der Mitglieder abhängig zu machen. Sähe man eine Zweidrittelmehrheit als verfassungsrechtlich geboten an, hätte dies zur Folge, dass es ein Drittel der Mitglieder plus Eins in der Hand hätten, Mitglieder in der Fraktion zu halten, mit denen die absolute Mehrheit nicht mehr kooperieren möchte. Der Mehrheit bliebe nichts anderes übrig, als von ihrem Austritts- beziehungsweise Neugründungsrecht Gebrauch zu machen. ${ }^{24}$

Da der VerfGH diese Frage offen lassen konnte, soll das Problem nicht weiter vertieft werden.

(3) Uneingeschränkte Zustimmung verdienen die Ausführungen des Gerichtshofs zu der materiell-rechtlichen Überprüfung eines Fraktionsausschlusses. Hier entfaltet der VerfGH die Fraktionsautonomie beziehungsweise -freiheit überzeugend, geradezu lehrbuchartig, und wendet die von ihm aufgestellten Grundsätze konsequent an. Vor allem die Belange der politischen Praxis, die bei den fraglichen Fällen oft auf zügiges Handeln angewiesen ist, um größeren Schaden abzuwenden, berücksichtigt er ausreichend, ohne den verfassungsrechtlichen Rechtschutz völlig leerlaufen zu lassen. Dies gilt in besonderem Maße insoweit, als die Ermittlung und die Bewertung des für den Fraktionsausschluss für erforderlich gehaltenen Sachverhalts den Fraktionen grundsätzlich selbst überlassen bleibt und der VerfGH eigene Ermittlungen ausschließt. Damit dürfte er etwas weitergehen als andere Verfassungsgerichte.

21 Dazu, auch zu den Begrifflichkeiten, ausführlich Bernd Grzeszick, a.a.O. (Fn. 12), S. 985, S. 988 ff.

22 Vgl. zum Beispiel Art. 42 Abs. 2 GG, dazu BVerfGE 112, S. 118, S. 140; Art. 88 Abs. 2 LV RP, dazu, auch zu den Ausnahmen Volker Perne, in: Lars Brocker / Michael Droege / Siegfried Jutzi (Hrsg.), a.a.O. (Fn. 3), Art. 88 Rn. 6 ff.

23 Vgl. BVerfGE 112, S. 118, S. 140 in Bezug auf den Grundsatz der Spiegelbildlichkeit der Ausschüsse; dazu auch Utz Schliesky, in: Hermann von Mangoldt / Friedrich Klein / Christian Starck, Kommentar zum Grundgesetz: GG, München 2018, Art. 42 Rn. 60.

24 So geschehen bei der AfD-Fraktion im Landtag von Baden-Württemberg: 13 von 23 Abgeordneten verließen die Fraktion, weil die Zweidrittelmehrheit nicht zustande kam. Vgl. „Fraktion in Stuttgart zerbrochen“, in: Stuttgarter Zeitung online vom 5. Juli 2016, https://www.stuttgarterzeitung.de/inhalt.afd-in-baden-wuerttemberg-fraktion-in-stuttgart-zerbrochen-meuthen-trittzurueck.978bfa8e-897f-44b2-804e-4320d94d3427.html (Aufruf am 22. Mai 2019). 